\title{
A Review of Empirical Studies on Transfer Pricing Manipulation
}

\author{
Shaif Jarallah*, Yoshio Kanazaki**
}

\begin{abstract}
This research surveys the recent surge of empirical studies on transfer pricing manipulation by multinational enterprises (MNEs), tax-motivated transfer pricing, particularly from the year 1990 to present. The review tackles transfer pricing income shifting behavior of MNEs from three different perspectives: taxation relationship with profitability, intrafirm trade, and foreign direct investment (FDI). There have been significant developments and contributions in this field, despite many limitations, mainly concerning the availability of micro-data in general, (specifically intrafirm trade data which allows capturing much of the heterogeneity which is dangling within inter-sectors), and the tax measurement issue. Yet, this area of study is still developing and promises more achievements.
\end{abstract}

Keywords: Transfer Pricing, Multinationals, Profit Shifting, Taxation.

Submission Date : 6/14/2011 Revision Date : 8/23/2011 Acceptance Date : 8/26/2011

* Graduate School of Economics and Management, Tohoku University, Sendai, Japan. E-mail: shaifj@hotmail.com

** Professor, Graduate School of Economics and Management, Tohoku University, Sendai, Japan. E-mail: kanazaki@econ.tohoku.ac.jp 


\section{Introduction}

There is currently a vast amount of transfer pricing research. Many theoretical approaches have attempted to address the transfer pricing issue from three different perspectives: economic, mathematical programming, and behavioural1). Hirshleifer (1956) was the first to explain in depth the economics of transfer pricing and to give suggestions on how to determine the transfer prices of goods and services between production and distribution segments of multinational enterprises (MNEs). The research summarized in this article focuses on the empirical studies of income shifting behaviour of multinational companies and the tax impacts on multinational companies' decisions, foreign direct investment (FDI) and the countries involved.

For a review of prior studies on income shifting and the corporate tax rates impact on FDI, we have referred to Hinse (1999), Newlon (2000), and Devereux and Griffith (2002); and primarily relied on behavioural studies in order to provide an unbiased analysis of the practices of MNE's.

The literature of tax-induced transfer pricing behaviour can be divided into three main groups of studies which are closely related to one another as illustrated in Figure (1).

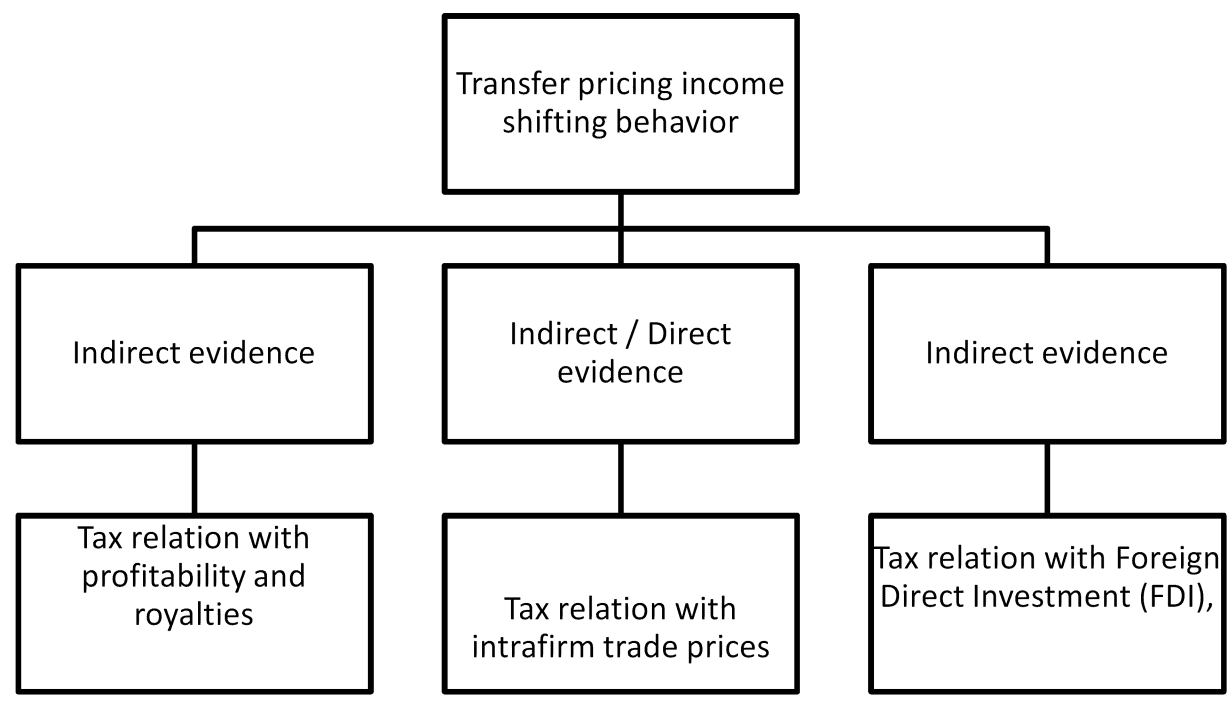

Figure 1.

Literature of tax-induced transfer pricing behaviour

1) See Abdul Khalil and Lusk (1974) 
- The first group of studies deals with the relationship between transfer pricing taxation and the profitability of MNEs. These studies usually apply to aggregate data, firm or affiliate level data and try to derive factors that affect transfer-pricing behaviour by analyzing the income and royalties of MNEs and their reactions to the tax variable changes.

- The second group of studies considers tax relation with intrafirm trade prices and analyzes firm- or product-level data on intrafirm trades. By comparing import and export prices to monitor tax incentives and the impact they have on MNEs transfer pricing activities, such studies usually provide direct evidence of the MNEs involvement in transfer pricing manipulations.

- The third group of studies focuses on corporate tax rate changes and the impact of transfer pricing manipulations on the capital flows and the location of the FDI. This group includes most of the European studies and utilizes aggregate data and company-and country-level data.

A graphical summary of the three groups of studies is included in Tables (1), (2), and (3)

\section{Definitions and MNE's Transfer Pricing Guidelines}

Transfer pricing is generally defined as the process of pricing goods and services between a multinational company and its subsidiaries and affiliates or related business entities across borders. Transfer pricing describes intrafirm trade of tangibles and intangibles (Plasschaert, 1979); a tactical procedure very often used by MNEs to minimize their worldwide tax burden and maximize their overall after-tax profits (Horst, 1971). MNEs are motivated to move profits from high tax jurisdictions to their subsidiaries situated in lower tax jurisdictions and tax havens (Grubert and Mutti, 1991; Harris et al., 1993; Hines and Rice, 1994). 


\begin{tabular}{|c|c|c|c|c|c|c|c|c|c|c|c|c|c|}
\hline 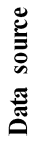 & 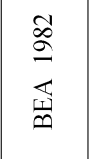 & 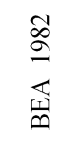 & 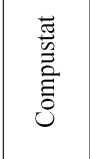 & 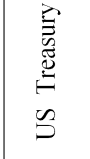 & 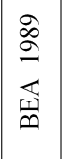 & 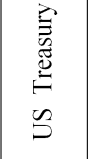 & 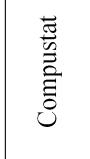 & $\begin{array}{l}\text { 志 } \\
\text { 言 } \\
\text { 言 }\end{array}$ & 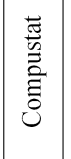 & 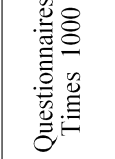 & 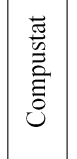 & 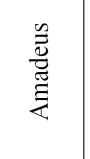 & 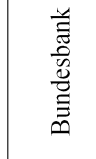 \\
\hline 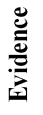 & 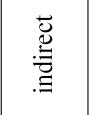 & 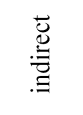 & 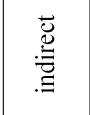 & 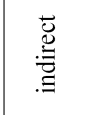 & 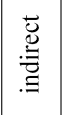 & 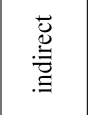 & 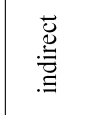 & 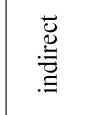 & 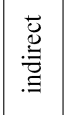 & 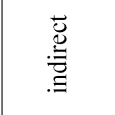 & 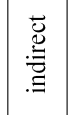 & 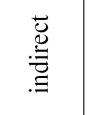 & 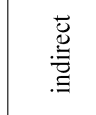 \\
\hline 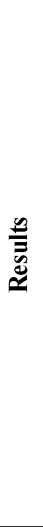 & 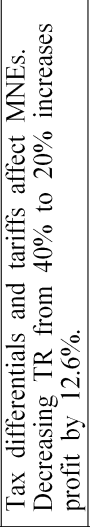 & 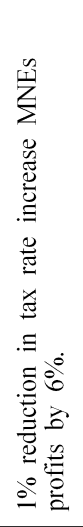 & 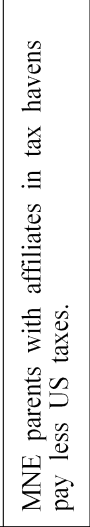 & 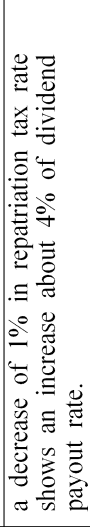 & 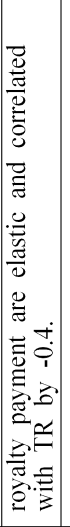 & 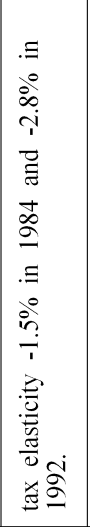 & 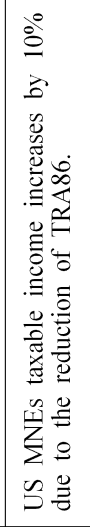 & 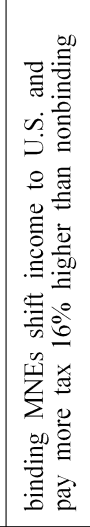 & 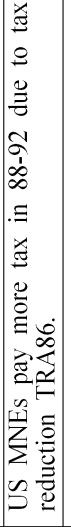 & 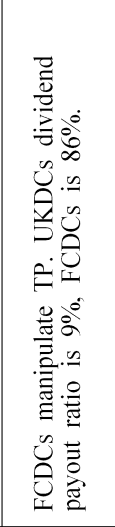 & 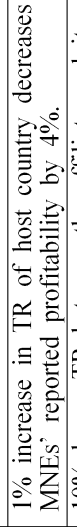 & 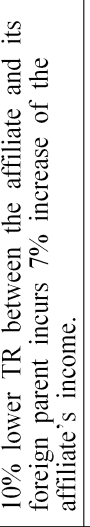 & 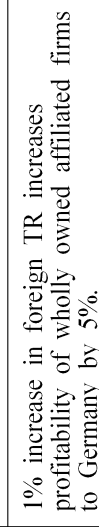 \\
\hline 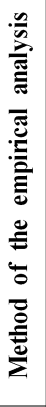 & 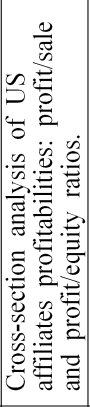 & 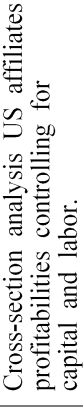 & 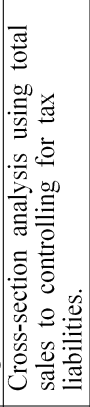 & 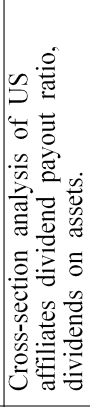 & 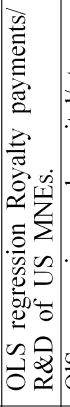 & 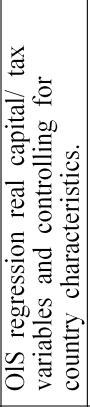 & 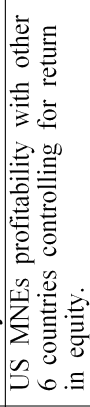 & 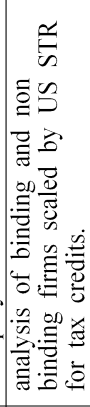 & 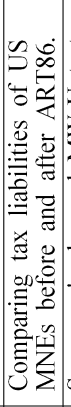 & 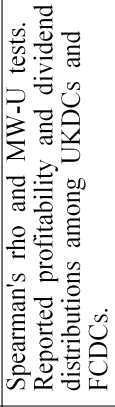 & 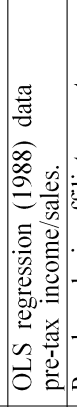 & 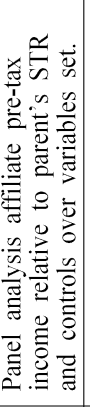 & 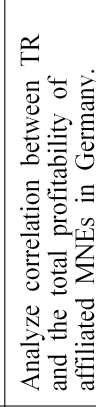 \\
\hline 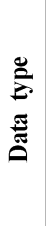 & 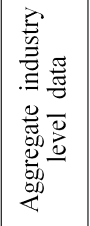 & 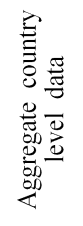 & 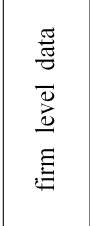 & 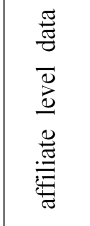 & 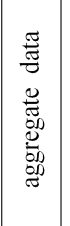 & 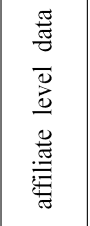 & 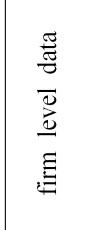 & 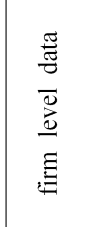 & 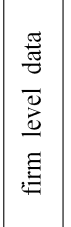 & 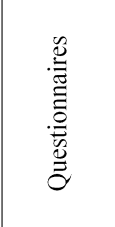 & 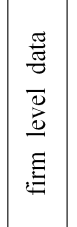 & 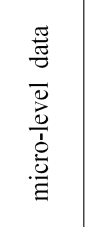 & 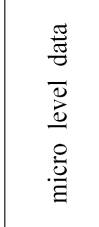 \\
\hline 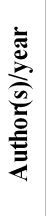 & 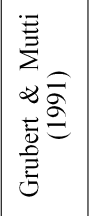 & 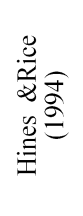 & 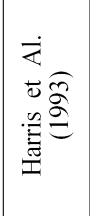 & 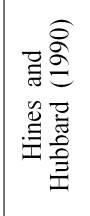 & 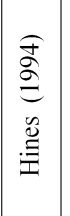 & 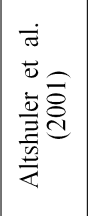 & 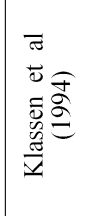 & 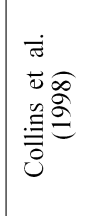 & 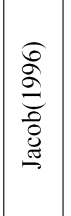 & 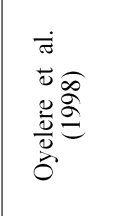 & 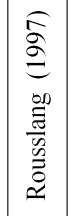 & 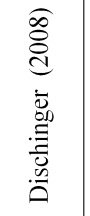 & 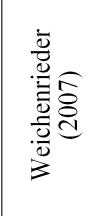 \\
\hline & \multicolumn{13}{|c|}{ 爮起 } \\
\hline
\end{tabular}




\begin{tabular}{|c|c|c|c|c|c|c|c|c|c|c|c|}
\hline & 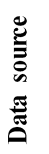 & $\begin{array}{l}\text { 㺃 } \\
\dot{\overrightarrow{0}} \\
\dot{0}\end{array}$ & $\begin{array}{l}\text { 吾 } \\
\dot{\vec{g}} \\
\dot{0}\end{array}$ & 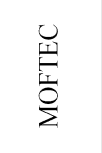 & 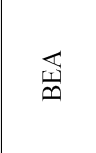 & 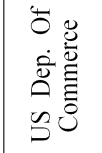 & $\vec{n}$ & $\stackrel{\tilde{m}}{n}$ & 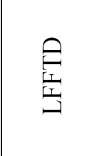 & 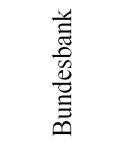 & 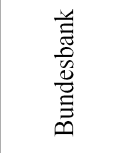 \\
\hline & 总 & 苞 & 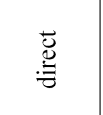 & 苑 & 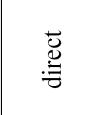 & 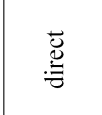 & 苞 & $\begin{array}{l}\overrightarrow{0} \\
\stackrel{\mathscr{J}}{=}\end{array}$ & 总 & 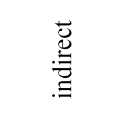 & 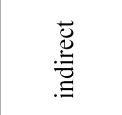 \\
\hline & $\frac{\mathscr{2}}{\underline{z}}$ & 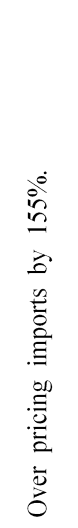 & 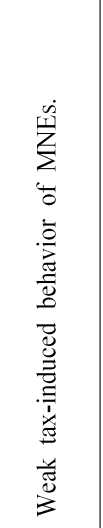 & 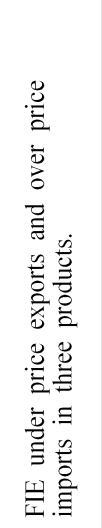 & 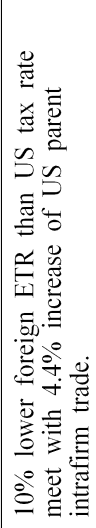 & 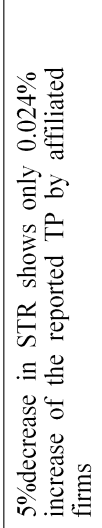 & 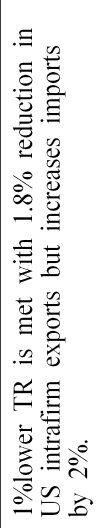 & 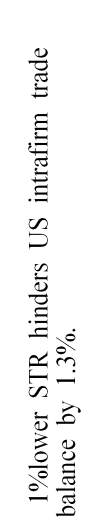 & 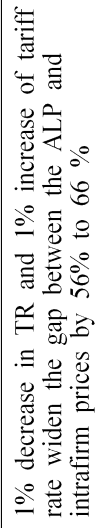 & 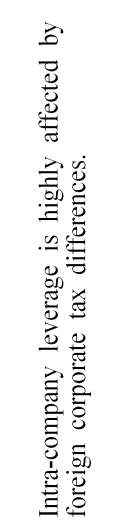 & 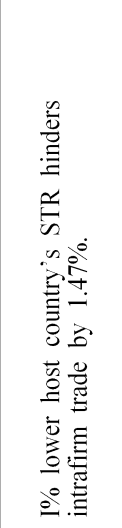 \\
\hline & 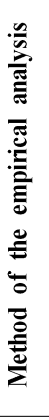 & 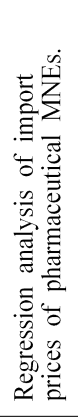 & 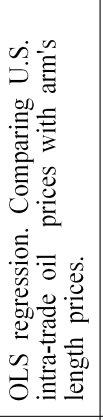 & 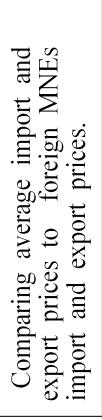 & 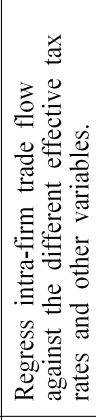 & 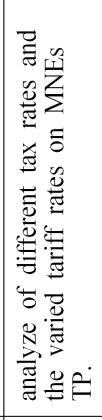 & 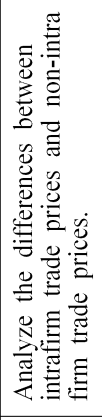 & 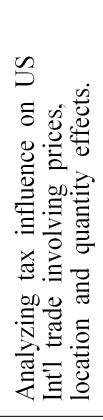 & 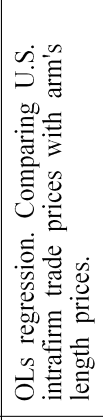 & 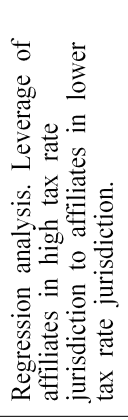 & 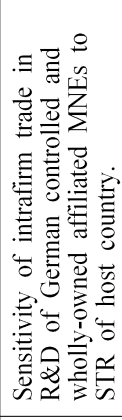 \\
\hline $\bar{\emptyset}$ & 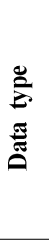 & 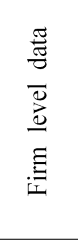 & 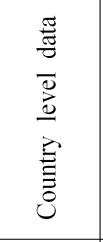 & 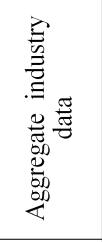 & 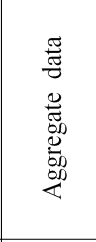 & 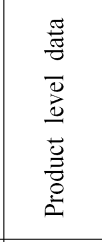 & 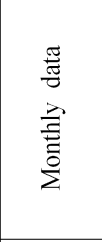 & 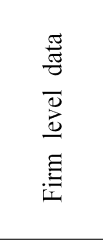 & 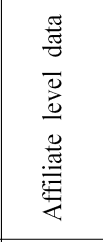 & $\begin{array}{l}\frac{\pi}{\tilde{J}} \\
\overline{0} \\
\overline{0} \\
\frac{0}{0} \\
\frac{3}{3} \\
\frac{3}{2}\end{array}$ & $\begin{array}{l}\frac{\pi}{\pi} \\
\overline{0} \\
\overline{0} \\
\frac{0}{0} \\
\frac{0}{0} \\
\frac{0}{2}\end{array}$ \\
\hline 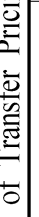 & 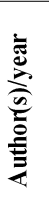 & 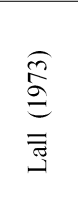 & 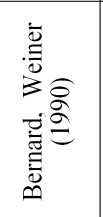 & 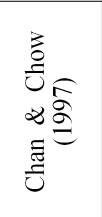 & 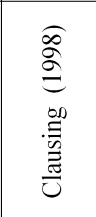 & 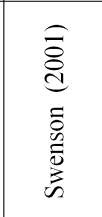 & 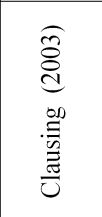 & 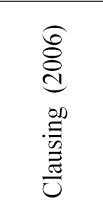 & 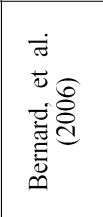 & 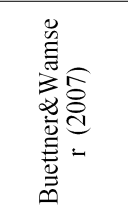 & 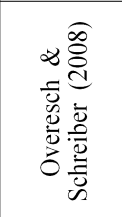 \\
\hline & 言 & \multicolumn{10}{|c|}{ 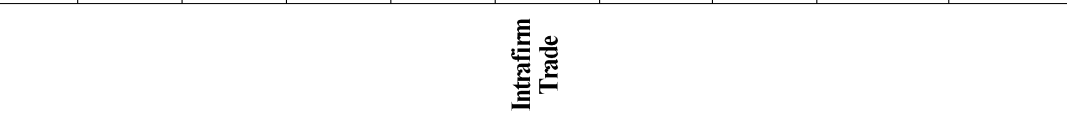 } \\
\hline
\end{tabular}




\begin{tabular}{|c|c|c|c|c|c|c|c|c|c|c|}
\hline & 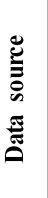 & 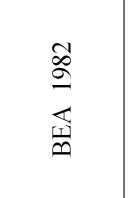 & 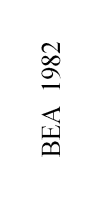 & 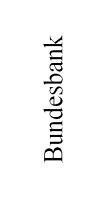 & 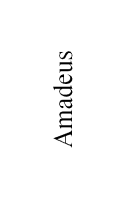 & 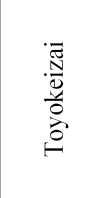 & 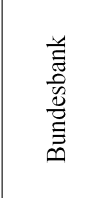 & 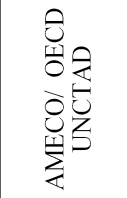 & 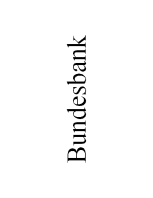 & $\tilde{\vec{n}}$ \\
\hline & 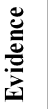 & 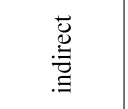 & 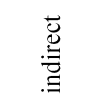 & 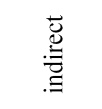 & 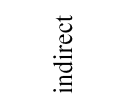 & 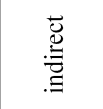 & 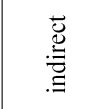 & 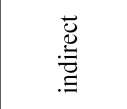 & 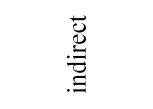 & 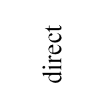 \\
\hline & 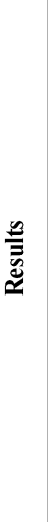 & 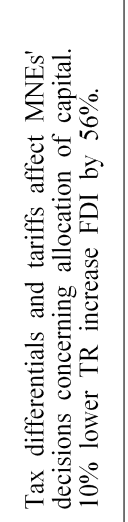 & 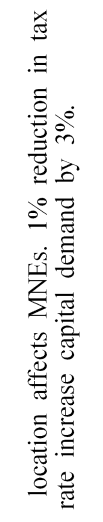 & 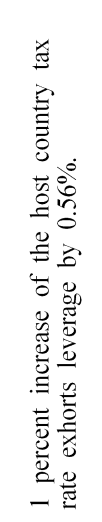 & 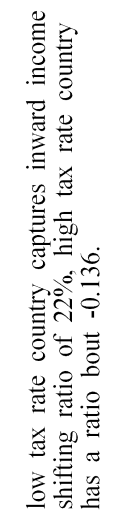 & 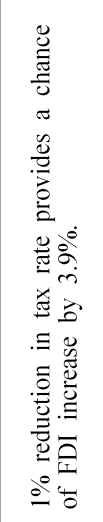 & 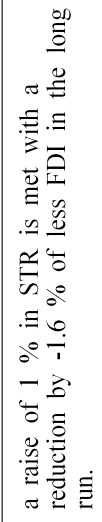 & 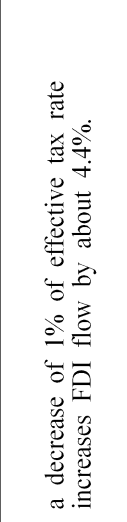 & 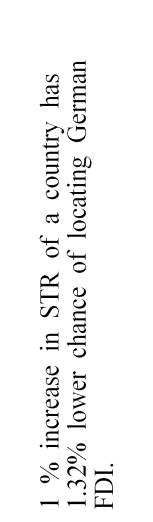 & 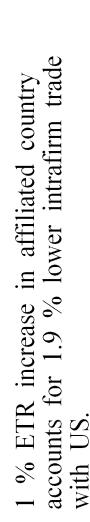 \\
\hline & 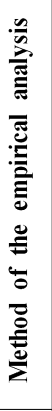 & 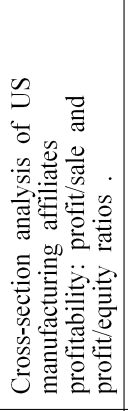 & 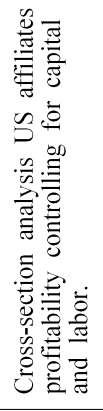 & 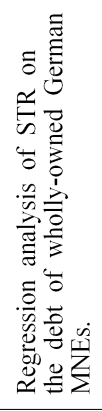 & 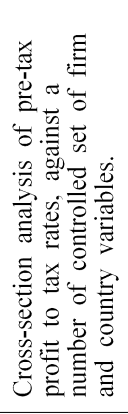 & 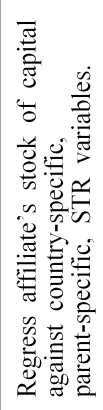 & 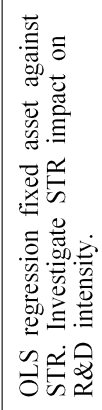 & 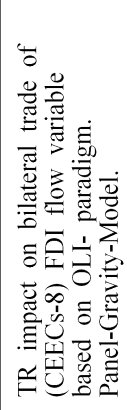 & 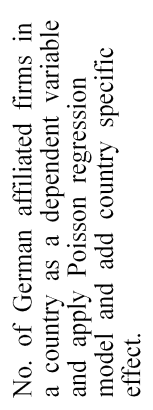 & 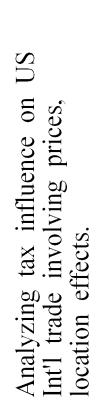 \\
\hline के & 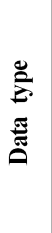 & 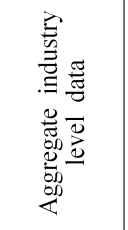 & 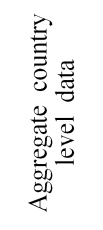 & 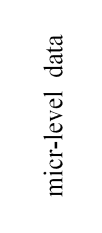 & 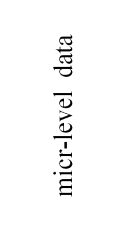 & 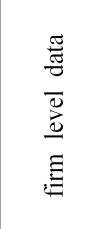 & 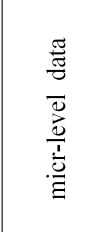 & 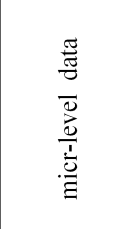 & 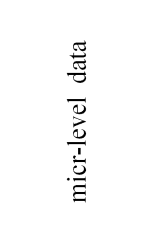 & 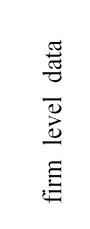 \\
\hline$\frac{\bar{a}}{\frac{\pi}{y}}$ & 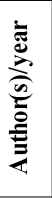 & 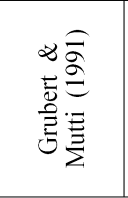 & 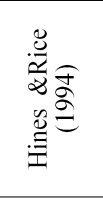 & 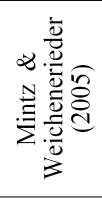 & 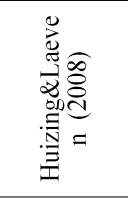 & 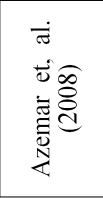 & 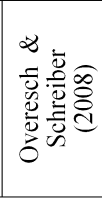 & 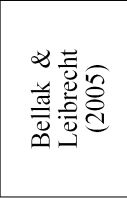 & 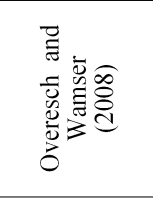 & 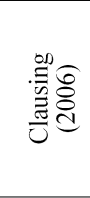 \\
\hline & छे & \multicolumn{9}{|c|}{ 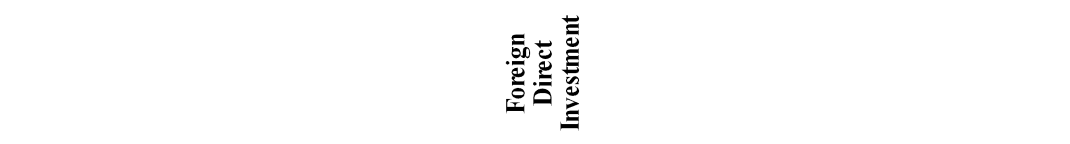 } \\
\hline
\end{tabular}


The Organisation for Economic Cooperation and Development (OECD) provides the commonly agreed-upon practice that governs and specifies international transfer pricing. The Arm's Length Standard (ALS) asserts that all business transactions between a MNE and its related parties be conducted similarly, in terms of prices, as other businesses transactions, as if they are set between unrelated parties, whenever transfer pricing manipulation exists (Article 9). The purpose of the ALS is to help solve the transfer-pricing problem by giving an estimation of the normal prices of traded goods or services among related parties. OECD member countries consider the OECD guidelines when assessing MNEs' transactions for taxation purposes. ${ }^{2}$ ) The specific guidelines include the comparable uncontrolled price method(CUP), the resale price method, the cost-plus method; the profit split method and the transactional net margin method(TNMM).

The CUP and resale price methods can be used to assess market prices of MNEs transactions of goods where prices can be observed. However, for intrafirm trade in intangibles or licensing and services, the CUP and resale price methods cannot be applied. The profit split method is used to determine the market price for such operations. The more unique tangibles and intangibles are, the greater the chance for MNEs to shift income (Feinschreiber, 2004). Other methods are mainly used to make close estimations of the market value of internally traded intangibles, via different profit ratio comparisons. Generally, countries will choose methods according to multiple criteria and MNEs will then try to adapt to this choice. 3 )

Some OECD member countries argue that the OECD has set its guidelines on the biases of the U.S. Internal Revenue Service (IRS) rules, particularly section 482 in the us income tax code. ${ }^{4}$ )

\section{Data and Tax Issues}

With increasing globalization, transfer pricing is one of the most important and complex tax issues being faced by the business world (Tyrrall and Atkinson, 1999). Transfer pricing is seen as one way to increase the profits of MNEs as well as for government entities to maximize tax collections.5) Most transfer pricing behaviour

2) Member countries are advised to adopt these guidelines to offer guidance to MNEs to apply the Arm's Length Standard when setting their transfer prices. These guidelines, however, are not obligatory. They do not measure up to binding rules, rather than just standards. For more comments on this issue see Baistrocchi (2004)

3) For more review on transfer pricing method selection, see, for example, Feinschreiber, R., (2004) Transfer Pricing Methods: An Applications Guide.

4) For more details see Eden et al (2001).

5) See Tyrrall, D. and Atkinson, M. (1999) for more information. 
literature places substantial importance on data and tax issues. The studies summarised here attempt to quantify the tax effects of both capital and the location of the investment. The type of data used--aggregate, country, industry, firm, macro or micro level--influences the results of each study.

\section{Limitations of the Data}

There appears to be a lack of suitable and reliable data in the field; the available data is mass-collected and does not separate MNEs from non-MNE or domestic from foreign companies. These limitations have complicated efforts to differentiate between industries and intrafirm trade practices from non-intrafirm trade practices. Most of the researchers so far have based their works on aggregate data, but this restricts modelling and prevents designing models focused more in identifying the used variables. In describing the weakness of the results obtained from the studies relied on aggregate data, Wolfson states: "much richness can be lost when our interferences about economic behaviour are necessarily restricted to economic aggregates," (Razin and Slemrod, 1990, p.200).

Empirical studies are divided into two groups which are related to the type of evidence they provide: either direct or indirect, (see Figure 1.) This is due to the restrictions and the limitations of intrafirm trade data. Consequently, because of the limitations of intrafirm trade data collection, most of the results of transfer pricing studies provide indirect evidence. More recently, some reliable data can be found in governmental organizations belonging to developed countries such as the U.S., Japan and Germany. These organizations include the U. S. Bureau of Labour and Statistics (BLS), Bureau of Economics and Analysis (BEA), Compustat, Japanese Ministry of Economy, Trade and Industry (METI) and the German Bundesbank. Very recently, there has been some quite detailed data available on multinational companies; although as prepared by Amadeus, Bureau Van Dijk, these reports focus on European multinational firms. In general, the availability of accurate micro data helps develop more sophisticated models and opens the door for researchers to investigate and explore the world of international transfer pricing with additional tools.

Recent studies have shown that MNEs often keep their transfer pricing arrangements undisclosed and privacy and confidentiality are prevalent (Borkowski, 1997). In addition, when governments and tax authorities investigate transfer pricing, they often do not make public the information regarding intra-firm trade, except for when it relates to transfer pricing adjustments. For example, the U.S. IRS required that a transfer pricing adjustment 
be made to the Nissan Company's 1975-1984 transactions amounting to approximately US\$1.85 billion (Borkowski, 1997, p.31). Additionally, the Glaxo Smith Kline Company was required to submit to the IRS more than US\$3 billion for necessary transfer pricing adjustments for the previous 16-year reporting period.

\section{Corporate Income Tax Rate Variables}

Almost all literature which we studied in connection with this research gives tax variables for corporate taxes a higher priority than other variables used in their analysis of transfer pricing or FDI studies, demonstrating the importance of tax issues for both government authorities and multinational firms. Governments depend heavily on tax revenues in order to sustain their welfare and development needs, while the primary concern of MNE's is to maximize profits. By employing various transfer pricing practices, MNE's are able to minimize tax liabilities and to some extent manage to obtain tax rebates, "despite anti-avoidance legislations and tax audit based on the arm's length principle," (Overesch, 2006)6).

Ernst \& Young (2007-2008) conducted a global survey on 850 MNE's across 24 jurisdictions and asked MNEs' tax managers to rank the importance of international transfer pricing taxation with regards to their operations. The survey results found that more than $74 \%$ of parent MNE's and $81 \%$ of subsidiaries declared that transfer pricing would be their prime concern related to tax issues within the next two years. The survey concluded that: "Globally, more parent companies are identifying transfer pricing as the most important issue that they face in comparison to other issues" (p12).

- Various tax rates, especially the statutory corporate tax rate and the effective corporate tax rate, are examined for their impact on MNE's transfer pricing behaviours and on FDI allocations. The statutory tax rate is used as a good tool to measure tax incentives for multinational companies in their transfer pricing arrangements; effective tax rates are regarded as an effective measurement of the tax effects on the FDI (OECD, 2000)7). According to the Tax Policy Centre, the

6) This is quoted from the "Non-Technical Summary" of the paper.

7) Clausing (2003), however, reaches a different conclusion, stating that neither effective tax rate nor the statutory tax rate that determines the "incentive to shift income between countries" rather the marginal tax rate which is considered as more proper and significant tool in measuring transfer pricing manipulations and income shifting. Yet, still, Clausing admits that even marginal tax rate is unable to capture the actually income corporate taxes paid. 
statutory corporate tax rate is defined as "the rate that is imposed on taxable income of corporations, which is equal to corporate receipts less deductions for labour costs, materials, and depreciation of capital assets... the effective corporate tax rate (ETR) measures the taxes a corporation pays as a percentage of its economic profit. Taxable income is less than economic profit when firms can exempt some income from their taxes, write off the cost of assets faster than their actual decline in value, or claim tax credits for certain business purchases. When taxable income is less than economic profit, a firm's effective tax rate is less than its statutory rate."(Tax Policy Centre: http://www.taxpolicycenter.org/briefing-book/key-elements/ business/statutory.cfm)

The tax differentials in different countries provide multinational companies the justification to participate in transfer pricing manipulation and shifting income between law and high corporate tax rates jurisdictions (CBO, 2005, pp.6-7). Tax rates are also determined by the industry sector and the size of the company (Nicodeme, 2001, pp.30-31). A summary of studies that examine sample sizes and the types of tax variables is available in Table 4. 


\begin{tabular}{|c|c|c|c|c|c|c|c|c|c|}
\hline 竞 & ๓. & 的善 & 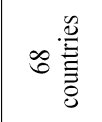 & 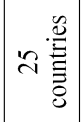 & 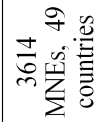 & 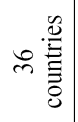 & 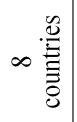 & ○葛 & 的蔨 \\
\hline $\begin{array}{l}\text { 受 } \\
\text { E }\end{array}$ & 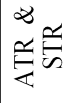 & 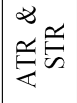 & $\underline{E}$ & $\stackrel{c}{5}$ & $\stackrel{\widetilde{v}}{\mathrm{~s}}$ & $\stackrel{\mathscr{E}}{\infty}$ & 螕 & $\stackrel{a}{n}$ & $\stackrel{\text { 喜 }}{\Sigma}$ \\
\hline 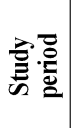 & $\stackrel{\mathscr{\sigma}}{\varrho}$ & $\stackrel{\mathscr{\varrho}}{\mathscr{O}}$ & 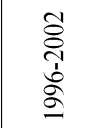 & ळे & ఠ్ণి & 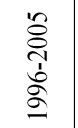 & 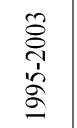 & 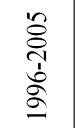 & 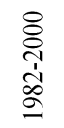 \\
\hline 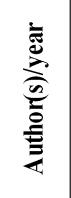 & 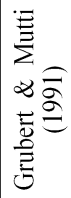 & 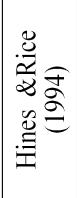 & 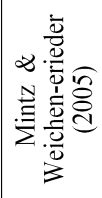 & 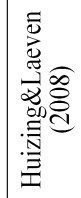 & 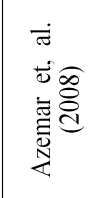 & 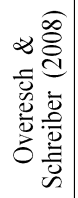 & 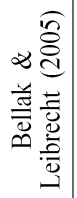 & 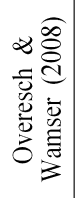 & 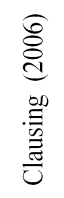 \\
\hline
\end{tabular}

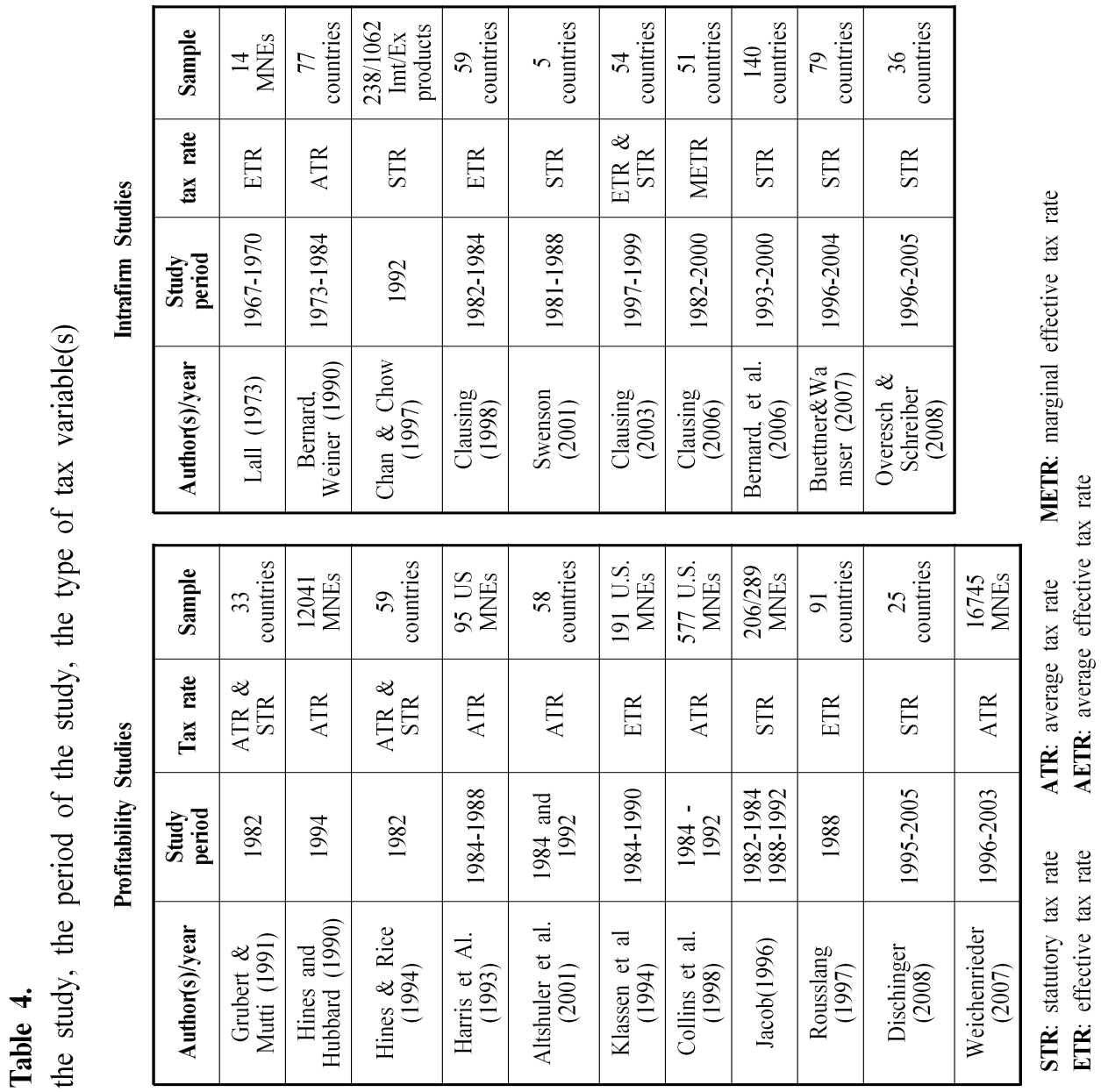


The most recent studies suggest the use of two different tax rates as the most proper method and offer close measurement of the taxes actually paid by MNE's. The first one is the Marginal Effective Tax Rate (METR). It is concerned with measuring incentives for marginal investment projects. The second measurement is the Effective Average Tax Rate (EATR). Dereveux and Griffith (1998) suggest that the EATR is the most effective way to measure the effects of taxation on MNEs' choice decisions on FDI location. ${ }^{8)}$ EATR is considered as the preferred measurement to capture the "distribution of tax rates for an investment project over a range of profitability," (Dereveux and Griffith, 1999, p. 37).

Most of the studies reviewed here use different corporate tax rate measurements or different calculations and their results do not reflect the real or actual paid taxes. Instead, they represent implicit taxes, which often diminish the complexity of the tax issues which were discussed earlier.

\section{Transfer Pricing Behaviours}

Previous transfer pricing behaviour studies have focused on three main areas: income shifting, intrafirm trade and FDI. Many researchers have pointed out the significant losses which the U.S. IRS experienced (R. Elitzur, J. Mintz, 1996). These losses compelled the IRS to make changes to its laws, such as Section 482, in the Tax Reform Act of 1986. Most of the American studies report on the changes after the passage of the 1986 TRA and compare tax effects on MNEs income shifting behaviour and FDI allocations before and after the 1986 reduction of the U.S. corporate tax rate.

When governments attempt to diversify their tax systems; this diversification adds to the complexity of the tax systems. MNEs attempt to minimize their tax burden by utilizing different strategies in order to build high taxation shelters. By taking advantage of tax differentials within countries, MNEs can under price their intrafirm exports to low tax jurisdictions and over price the intrafirm imports to high tax jurisdictions.

Due to the increase in global economies, the rise of economic integration and the expansion of the intermediate goods trade volume, governmental tax authorities who focus on international transfer pricing know that it is often manipulated by MNEs in order to minimize tax liabilities across countries (Grubert and Mutti, 1991; Harris et al., 1993; and Hines and Rice, 1994). The incentives for MNEs are to shift income in order to include

8) This paper does not attempt to provide methodologies how to compute the above mentioned different corporate tax rates. For more elaboration on this issue, refer to Dereveux and Griffith (1998) and Nicodeme (2001). 
reducing tax liabilities, avoid double taxation and move debt to jurisdictions with higher corporate tax rates (Harris et al., 1993, p.277). ${ }^{9}$ )

Intrafirm trade, the opposite of arm's length trade, involves trade in tangible and intangible goods and services. Approximately one-third of the transactions of multinational companies involve intrafirm trading (Eden, 2001), ${ }^{10)}$ affecting various economic indicators such as the balance of payments' figures, national income, and the prices of imports and exports (Eden 2000 p.7). In the United States, intrafirm trades represent more than one third of exports and 52 percent of imports. U.S. MNE imports and exports are primarily transactions between parent firms and their foreign affiliates (Zeile 1997, pp.25-27), although 70 percent of the U.S. imports are from Japanese MNEs (Bardhan and Jaffee 2004, pp.3-10).

Different incentives are offered by governments for MNEs to set up their businesses (Leitch and Barrret, 1992) knowing that they have to compete with different countries for FDIs and income taxes. Weichenrieder (1996) finds that some companies seek to attract MNEs by setting up tax havens. Within two decades, Ireland, for example, facilitated foreign direct investment by making it attractive for over 1,017 MNEs to base their businesses in that country. ${ }^{11)}$ While MNEs can create positive economic developments through foreign direct investment activities and development, the FDI also may severely hinder a government's ability to receive a legitimate share of taxable income. This may be due to the manipulating and shifting of financial sources and production across jurisdictional borders. A study by Gersik in 2001 showed that it is indeed difficult for governments to accurately measure MNEs' taxable income.12) MNEs have complex intrafirm trade activities within related parties, making it difficult to track actual transactions and resulting income. Several researchers have found that multinational companies sell goods and services to affiliates at non-market based prices, and then submit lower transfer prices to the taxing jurisdictions. ${ }^{13)}$

\footnotetext{
9) See also T. A. Gersik (2001). Buettner and Wamser (2007) discussed bellow.

10) It has been argued that nowadays intrafirm trade is representing more than half of the transactions globally traded, see Thomas C. Pearson (2008), pp.5-6.

11) Weichenrieder (1996, p. 41)

12) For more review on this issue, see T. A. Gersik (2001).

13) Grubert and Mutti (1991), Harris et al (1993), and Hines and Rice (1994) reviewed below. Also, see J. Edwards and M. Keen (1996).
} 


\section{Summary of the Studies}

The remainder of this article is a brief summary of studies of the empirical evidence of transfer pricing manipulations. The information listed here can only briefly list specific studies and their results. The studies are grouped according to the areas which were previously outlined.

Two earlier studies that used government data to examine transfer-pricing manipulation MNEs were the study of Colombian MNEs15 of pharmaceutical, rubber and electrical sectors, conducted by Lall (1973), and the study of oil prices of US petroleum MNEs, conducted by Bernard and Weiner (1990). Lall analyzed the prices of imports of pharmaceutical multinational firms within the period 1967-1970. The results provided evidence that MNE manipulation was detected. He found that the majority of the wholly owned foreign firms' imports were overpriced by an average of 155 percent. Bernard and Weiner used data from the Energy and Information Administration (EIA), from 77 countries, during the period 1973 to 1984 . They studied the prices of multinational oil companies and compared them with the prices of other unrelated companies. The results showed that oil companies were less involved in tax-motivated transfer pricing and that there was no significant correlation between transfer pricing differentials and the corporate tax rate. Their study found "very weak support for the influence of an effective income tax rate on transfer prices between affiliated parties." They credited this to the effectiveness of IRS enforcement. Other prior studies of US MNEs oil prices, however, had found significant relationships between transfer pricing income shifting and corporate income tax rates. ${ }^{14)}$

\section{Profitability studies}

This set of studies investigates the consequences of corporate income tax rates on MNEs' activities and attempts to measure how much tax rates affect MNEs reported profitability. These studies answer the question: What are the other variables utilized by studies which analyse MNE profitability?

Two studies, Grubert and Mutti (1991) and Hines and Rice (1994), used the same

14) Jenkins and Wright (1975) investigated oil industry during 1966 and 1970. They provided evidence of taxation effect on oil industry that the profitability of US oil companies depending on their affiliates locations and taxation. They realized that US oil companies barely pay tax to US government. They transfer their profits to producing crude oil countries. 
benchmark data from a 1982 survey of U.S. (BEA) and focused on the tax impact on the profitability of MNEs and the location of capital. Grubert and Mutti (1991) examined the relationship between US MNEs after-tax profitability and corporate tax rates. They suggested that corporate tax rates impact on reported profits are highly significant. Grubert and Mutti suggested that a reduction in the corporate tax rate from 40 percent to 20 percent would also result in an increase from 14.2 percent to 20.7 percent of the after-tax rate of the return on equity, Harris et al. (1993, p.278). These findings are criticized by Hines and Rice, who stated that the measurements of profitability of the former study lacked accuracy (p.58). While Grubert and Mutti constructed their cross-section data from 33 countries where US manufacturing foreign affiliates were situated, Hines and Rice utilized a larger aggregate cross-section data from 73 host countries, to test whether US MNEs shifted profits and production activities to low-tax rate countries, out of which 41 countries were tax havens (p.164).15) Both studies confirmed that corporate tax rates had a significant influence on U.S. MNEs' profit shifting behaviour, although the data which they used was before the tax reform act (TRA 86), which scrutinized transfer pricing.

Harris et al. (1993) used five-year firm level data to discover the viability of income shifting evidence on transfer pricing activities of 95 U.S. MNEs during the period from 1984-1988. The researchers examined the relationship between U.S. multinational firms' tax payments and the locations of the foreign jurisdictions with different tax rates. High tax rates countries like Japan, Canada, and west Europe were compared with low tax rates jurisdictions such as Ireland, Tax Havens and the Four Dragons. Harris and others used total sales or assets as variables to determine firms' tax liabilities. They discovered that US tax payments by MNEs in low tax rate jurisdictions were lower than those in high tax rates jurisdictions. The Harris researchers concluded that U.S. MNEs were actively involved in transfer pricing manipulations and the results were strongly consistent with tax-induced income shifting, both from U.S. manufacturing subsidiaries in high tax rate countries to subsidiaries in low tax rate countries and from U.S. parent companies to low tax rate subsidiaries.

While studying the sensitivity of U.S. MNEs to the global changes of corporate tax rates over the period 1984 to 1990, Klassen, Lang, and Wolfson (1993) utilized the Compustat data of 191 U.S. MNEs. They examined the incentives of these changes in tax rates, especially the reduction after the U.S. TRA 86, to the geographic income shifting behaviour of U.S. MNEs. Their sample data included two groups, U.S. operation firms and non-U.S. operation firms. They concluded that income shifting from Canada to the U.S. increased in 1986, when the Canadian corporate tax rate was higher relative to the U.S. tax

15) They test for both GDP and population variables and they find that log population has explanatory power to explain tax rates more than the $\log$ GDP variable 
rate. During the previous reporting period of 1984-1986, income shifting primarily occurred from Europe and other regions to the U.S. In general, reported taxable net income by U.S. operations increased by 10 percent compared to the non-U.S. operations during the reporting period. The results of the Klassen study are also consistent with previous findings.

Altshuler, Grubert, and Newlon (2001) examined the effective tax rate impact on the business allocation decisions of U.S. manufacturing MNEs decisions. They used data from the U.S. Treasury for two time periods--1984 and 1992 - and utilized information from Form 5471 to calculate the capital of affiliated MNEs in 58 countries. While controlling for certain tax and country variables, Altshuler et al. found that the elasticity of capital to tax rate differences increased from -1.5 in 1984 to -2.8 in 1992 due to the tax reduction after TRA 86.

Jacob (1996) randomly selected U.S. firms' data from Compustat during two-time periods- before and after 1986- to reflect the impact of transfer pricing rules and corporate tax rate changes within these periods. He targeted the influence of tax rate differences and time intervals on income shifting behaviour of U.S. MNEs. He found that more U.S. multinational firms with considerably large amounts of global intrafirm sales paid lower global tax liabilities during both periods and paid lower corporate taxes within the U.S. during 1982-1984. However, multinational firms paid higher U.S. corporate taxes during 1988-1992. He concluded that MNEs with higher levels of intrafirm trade had higher incentives to shift income through transfer pricing because tax rates decreased after TRA86. Jacob's proof also contributed generally to the tax-motivated MNEs transfer pricing practices.

Collins, Kemsely, and Lang (1998) used Compustat data and constructed a pooled sample of firm-level data of 577 MNEs, over the period 1984 to 1992. They calculated the average of both rate of return and the effective tax rate of U.S. foreign affiliates scaled by the U.S. statutory tax rate. Collins et al. grouped firms on the basis that if an affiliate faced a foreign average tax rate higher than the U.S. statutory corporate tax rate, then it became binding to receive U.S. foreign tax credit payments; if the company's situation was vice versa then it became nonbinding. They figured out that binding companies were more induced to shift income to the U.S., to obtain tax credit, and report higher foreign taxed income while nonbinding ones reported lower foreign taxed income. Collins and others remarkably proved that an increase of 16 percent of foreign income was shifted into the United States. They referred this increase in repatriation to the initiatives of the IRS in 1986 (TRA86), which reduced the corporate tax rate from 46 percent to 34 percent.

Hines and Hubbard (1990) used affiliate-level data of most of the large U.S MNEs from the U.S. Treasury, over the year 1984. They investigated the U.S. MNEs transfer pricing manipulation through the foreign affiliates' dividend payout rates to their parent 
companies. They discovered that most U.S. affiliated companies paid very low taxes to the U.S. government; they preferred to pay dividends to their parent companies rather than royalties, interest, and rent. This is consistent with tax avoidance. Hines and Hubbard suggested that a decrease of $1 \%$ in the U.S. repatriation tax rate showed an increase of about $4 \%$ in the dividend payout rate. They stated that affiliates paid more dividends to parent companies when parent companies had excess tax credits. They contributed the low dividend taxes to the tax credit system and the deferral of taxes on repatriation, which motivated MNEs to avoid paying dividends.

Another study by Hines (1994) analyzed aggregate data, of the BEA survey in 1989 of royalty payments, of both foreign affiliates to their U.S. parents and U.S. companies to their foreign parents within 41 countries. Hines found that royalty payments are elastic and negatively correlated with royalty tax rates by -0.4 ; reducing tax rate increases of royalty payments, while increasing tax rates by one-fold decreases royalties by 40 percent. The author interpreted this result as evidence of MNEs manipulation of transfer pricing to minimize their global tax burdens.

Following similar approaches of Grubert and Mutti (91) and Hines and Rice (94), Rousslang (1997) analyzed Compustat data (1988) to detect the U.S. manufacturing MNEs tax-induced profit shifting behaviour. He reached similar results achieved by both the above-mentioned studies. Again, TRA86 was regarded as a reason for the reduction of U.S. MNEs transfer pricing manipulations. The average effective tax rate was considered in the regression analysis as a better measurement for transfer pricing incentives because the statutory tax rate failed to capture foreign tax holidays and other deductions. Rousslang concluded that a 1 percent increase in the corporate tax rate of a host country decreases MNEs' reported profitability by 4 percent.

Oyelere (1998) investigated the relationship between MNEs' reported profitability and dividend distributions of both UK-based foreign controlled affiliates and UK controlled multinationals. The data collected through correspondences asked companies to provide financial statements. Data, during the period of 1992-1993, on 72 companies, 32 of each sample compared with the total assets, were constructed. Total asset value was used to measure the capability of firms, performance was measured by profitability, and dividend distribution was used to measure post performance. The regression analysis revealed a variation of profitability and dividend distribution between the two samples. The profitability of foreign controlled affiliates, showed low performance, and was represented by only 66 percent of UK controlled companies. However, post performance, against expectations, showed that foreign controlled affiliate's dividend payout ratio was significantly higher than UK controlled multinationals. UK MNEs payout ratio represented 9 percent for each year while the foreign controlled MNEs payout ratio represented 
respectively 86 percent in 1992 and 36 percent in 1993 . He remarked that manipulation of foreign controlled affiliates by under pricing intrafirm trade was the reason for lower performance and that income shifting could be traced from the dividend payout distribution of foreign affiliates compared to their local counterparts.

Weichenrieder (2007) used micro level data from Direct Investment (MiDi) of Bundesbank to investigate profit-shifting behaviour of foreign affiliated MNEs in Germany over the period 1996 to 2003. He found that there was a significant correlation between the parent firms' corporate tax rates and the total profitability of affiliated MNEs in Germany.

Overesch and Wamser (2008) also applied firm level data from (MiDi) of Bundesbank to look into tax rate effects on German MNEs financing decisions in ten Eastern European countries. They used total liability to total capital ratio as dependent variables and control for other variables, country, firm specifications and time effect. They found that high tax rates were associated with increased debt demand.

Dischinger (2008) found significant evidence of tax avoidance and income shifting behaviour by employing more accurate explanatory tax measure variables. The researcher employed micro-level panel data obtained from Amadeus on 14,077 multinational subsidiaries located in 25 European countries; focusing on the years from 1995 to 2005. Dischinger found strong negative relationships between affiliates' tax rate differentials associated with their foreign parent corporations' tax rates and affiliates total income. Dischinger's findings concurred with those of other researchers (Overesch, 2006; Weichenrieder, 2007) who also documented indirect evidence of income shifting behaviour while controlling for the ownership share of the firm. He found that tax differentials were strongly correlated with the ownership share of the firm.

\section{Intrafirm Studies}

Intrafirm studies focused primarily on the influence of the different income corporate tax rates of countries with respect to MNEs' transfer pricing income-shifting activities when conducting intracompany trade. Unlike many studies, which elaborated on transfer pricing manipulation by comparing tax rates with profitability or royalties, the second approach is dealing with transfer pricing behaviour through intrafirm trade data, import and export prices. These studies seek to answer various questions such as: 
- What is the relationship between corporate tax rates and intrafirm trade of import and export prices?

- To what extent do MNEs intercompany activities shift income from the parent country to the host country and vice versa?

- How does intrafirm trade encourage MNEs to become involved in profit shifting practices, and manipulate the prices of internal transactions?

- How significantly is intercompany financing (leverage) affected by foreign corporate tax rate differentials?

Studying the reporting period of 1992, Chan and Chow (1997) examined aggregate data of import and export products in China by both foreign multinational companies and domestic companies over the year 1992. The data was collected from the Ministry of Foreign Trade and Economics Corporation (MOFTEC) and the Chinese Foreign Economic and Trade Statistical Yearbook. By comparing average 1992 MNE import and export prices in China, Chan and Chow found that the foreign MNEs import prices were 11 percent higher than that of domestic companies. They also unexpectedly discovered that the export price of foreign firms was also 11 percent higher than that of similar Chinese firms. Although the findings seem to dispute the presence of transfer-pricing income shifting behaviour by foreign MNEs, the researchers concluded that transfer pricing manipulation was present in specific industrial sectors: mainly electronics, garments and plastic products. In those sectors, foreign MNEs under price exports and over price imports. The aggregated data used in this study did not utilize controls that separated data between intrafirm trade and non-intrafirm trade.

In a later study, Clausing (1998) examined aggregate intrafirm data taken from BEA, a survey of US direct investment overseas from 1982 to 1994. According to time- and country-controlled tax rate variables and focusing on intra-trade between US MNEs parents and their scattered affiliates over 59 countries, the researcher used intrafirm trade flow as a dependent variable and regressed it against the different effective tax rates and other variables. Clausing found that U.S. intrafirm trade was underpriced when exports took place with low tax countries while overpricing occurred on imports to high-tax countries; agreeing with previous studies which concluded that tax-induced transfer pricing is aggressively practiced by U.S. MNEs.

Swenson (2001) employed product-level data from the U.S. Census Department to study transfer-pricing manipulation among U.S. MNEs during 1981-1988 when many tax rate and tariff changes took hold in the global economies. Studying the prices of U.S. 
imports from five of America's largest trading partners, Swenson found a very weak correlation between corporate tax rate reductions and reported transfer prices. Swenson concluded that this weak finding was the result of the data including non-intrafirm trade.

Clausing (2003) continued her extensive research on this topic by using monthly data on US international trade prices from the BLS, Index Pricing Program (IPP) from 1997 to 1999. Separating intrafirm trade from arm's length trade, she found that the tax rates of countries have a substantial influence on intrafirm trade transactions: the lower the tax rate of a country, the lower the prices of the exports of US intrafirm trade, and the higher the import prices were of US intrafirm trade relative to non-intrafirm trade. Eden (2001) found inconsistencies in this study charging that although Clausing used BLS (IPP) data during the above-mentioned period; the separated intrafirm trade data was not added until February of 1998.16)

Bernard, Jensen, and Schott (2006) studied a unique data set from the U.S. Census Bureau, (LFTTD) database17), which recorded all U.S. international transactions traded within 140 countries; for the time period between 1993 and 2000. Bernard et al. investigated the differences between export prices for intrafirm trade and export prices for arm's length trade. In their OLS regression, they employed the difference between arm's length prices and related party prices as a dependent variable and control for country and firm characteristics such as statutory tax rate differences, tariff rate variations, firm size, and export share. They found that a decrease in the tax rate and an increase of the tariff rate widened the difference between the arm's length prices and the intrafirm prices. Bernard's findings were also consistent with other studies of intrafirm transfer pricing manipulation of U.S. multinational firms.

Clausing (2006) examined the influence of tax rates on U.S. international trade flows, including price, quantity, and location variables, utilising the BEA firm-level database information on 51 countries from 1982 to 2000. She found that prices determined by US intrafirm trade balances were significantly correlated to the tax rate. She further found no significant impacts on intrafirm trade in high-tax countries; but low-tax countries were more greatly affected. Clausing attributed this to the fact that low-tax countries were more likely to invest in intrafirm trades.

Overesch (2006) examined information during the time period of 1996-2003 from the MiDi micro-level panel database from the Deutsche Bundesbank on all German MNEs located in 31 countries. He used the itemized balance sheet in the financial accounts of MNEs and variables that affected intrafirm trade. He found that the higher the invested

16) For more review concerning this issue and BLS (IPP), see Eden (2001).

17) Linked/Longitudinal Firm Trade Transaction Database 
capital in an affiliate, the higher the intrafirm sales in that affiliate. Additionally, Overesch concluded that shifting behaviour intensified with the increase of ownership shares in the company.

Buettner and Wamser (2007) also used the German Multinationals (MiDi) firm-level database provided by Bundesbank in 79 countries for the nine-year period from 1996 to 2004. Focusing on the total liability of affiliated MNEs, they tested whether the intrafirm debt of MNEs was used to shift profits from affiliates in high tax rate jurisdictions to affiliates in lower tax rate jurisdictions. The results confirmed that the leverage among intra-companies was significantly affected by foreign corporate tax differences.

The final study summarized in this area was conducted by Overesch and Schreiber (2008) who evaluated the MiDi micro database and showed that tax sensitivity to intrafirm trade could be highly affected by the increase of R\&D intensity of MNEs. They found that the tax rate was highly correlated to a high rate of $R \& D$ activity in a firm. In a similar finding by Clausing (2006), the researchers also concluded that a reduction in the host country's statutory tax rate hindered intrafirm trade.

\section{FDI Studies}

Studies on transfer pricing behaviour demonstrate the impact of taxation on FDI, and MNEs study the taxation policies of various countries since those practices can greatly affect their after-tax net profitability. This third group of studies focuses on how tax rates influence real activities of MNEs and provide evidence of MNEs' sensitivity to tax incentives. The following questions will be examined:

- What is the relationship between the rate of corporation tax in a country and the rate of foreign investment?

- To what extent are MNEs influenced by tax motivations offered by countries to locate their investments?

- How do corporate tax rates determine other factors such as leverage, R\&D and how do they affect the foreign investment decisions of MNEs? 
Several researchers studied the effects of corporate tax rates in the U.S. on the demand for capital. Grubert and Mutti (1991) found that a decrease in the corporate tax rate in a country from 20 percent to 10 percent would increase demand for capital in that country by 65 percent. Similarly, Hines and Rice (1994) concluded that a 1 percent reduction of the corporate tax rate would increase the FDI by 3 percent. Both studies provided evidence that corporate tax rates have significant influence on U.S. MNEs' decisions on how to allocate their businesses.

After data sources such as Bundesbank and Amadeus became more widely available, several studies focusing on European companies were submitted. These studies provided more analysis and more highly significant results due to the greatly detailed data. Most of these studies investigated the logic and motivation pertaining to the decisions of MNEs for FDI.

Mintz and Weichenrieder (2005) examined the FDI activities of German MNEs over the period 1996-2002, based on the hypothesis that high corporate tax rates encourage MNEs to finance intracompany operations in high tax jurisdictions with debt to obtain tax shields. They concluded that German MNEs were motivated to locate their wholly owned subsidiaries in low tax rate countries and depended heavily on intercompany leverage to obtain benefits from leverage interest rate differentials and tax-exempt financing.

Azemar, Corcos, and Delios (2008) examined firm-level data of 3614 Japanese MNEs scattered in 49 countries; testing the influence of tax rates on wholly owned and high R\&D affiliates and joint ventures low in R\&D. They found that large Japanese parent corporations with high $R \& D$ activities were more highly influenced by foreign tax policies than low R\&D affiliates. Study results suggested that Japanese overseas capital investment in emerging countries was affected by foreign corporate tax rates.

A study by Overesch and Schreiber (2008) provided evidence that FDI is less sensitive to the tax rate of host countries when the R\&D activities of the MNE are concentrated in that country. The researchers used debt financing as an alternative to profit shifting and employed the debt ratio as a dependent variable and control for other firm and country specifications. They suggested that a rise of 1 percent in the statutory tax rate is met with a reduction by -1.6 percent of decreased FDI in the long run.

Another study by Bellak and Leibrecht (2005) focused on the influences of FDI on Central East European Countries (CEECs). They examined the impact of corporate tax rates on the bilateral trade of eight host countries (CEECs-8) with seven home countries over a nine-year period from 1995-2003. The FDI flow was used as a dependent variable based on the OLI paradigm (Ownership, Location and Internalization). Bellak and Leibrecht concluded that countries with high corporate tax rates are the least attractive for FDI and that MNEs strongly consider the tax rates of different countries when making decisions 
about FDI.

Overesch and Wamser (2008) followed a similar approach with their study which focused on the tax impacts on FDI. Utilising German Central Bank data on German controlled firms. They integrated the number of German affiliated firms in a country as a dependent variable and applied the Poisson regression model and added country specific variables. They concluded that the corporate tax rates of host countries in Eastern Europe negatively affected the decisions of MNEs about where to establish their FDIs. Overesch and Wamser stated that their "results were remarkable because they suggested that taxes were the only direct policy instrument which was relevant for the investment decision" (p.20).

In the final study reported in this article, Huizing and Laeven (2008) used data sourced from Amadeus on MNEs from 25 European countries. The selected MNEs all had at least one subsidiary. The researchers constructed a model that tracked the income shifting behaviour of European MNEs; including variables such as tax differences and income shifting between and among the parent and affiliate companies. The study found that income shifting moved inward within lower tax rate jurisdictions and outward within higher tax rate jurisdictions. These results also reflect how many countries justify attempts to maintain a competitive tax position in order to attract more FDI activities by reducing their corporate taxation policies.

\section{Conclusion}

The purpose of this article is to synthesize the extensive amount of existing studies on transfer pricing manipulation of MNEs and the allocation of FDI while also presenting some of the difficulties experienced by researchers in this field. The primary findings of the research summarised in this article conclude that corporate tax rates are greatly affected by the transfer pricing behaviours and decisions of MNEs as they make allocation decisions. Most of the literature concludes that the reduction of taxes and the simplification of governmental taxation systems encourage foreign direct investments within countries. MNEs place great importance on transfer pricing taxation practices and consider those policies as conduits through which increased profitability can be achieved.

Other factors, such as exchange rates, inflation, and capital movement, also affect MNEs transfer pricing behaviour, although most of the research mentioned in this article seeks to establish the measurable impacts of corporate tax rates on MNEs and FDIs. 
This article also attempts to expose possible problems in some of the studies, including:

- Measurements of tax rates are still imprecise and unreliable. Models capable of calculating and measuring the right effect of tax rate on the profitability and the location of capital have yet to be developed.

- Data availability is restricted and the aggregate and micro data used by most of the researchers is usuallt only easily available in relatively few developed countries.

Although many of these issues could seriously impact the findings of the studies, many improvements have been made in the quality of the literature on transfer pricing; including the theoretical models and estimation prototypes. This article is presented to the readers and researchers in the hope that future studies will produce more sophisticated and accurate models on which further results can be based. 


\section{References}

Altshuler, R., H. Grubert, and Newlon, T. S. (2001), "Has US Investment Abroad become More Sensitive to Tax Rates?”, in J.R. Hines Jr. (ed.), International Taxation and Multinational Activity, Chicago: University of Chicago Press, pp. 8-32.

Baistrocchi, Eduardo A., (2004), "The Arm's Length Standard in the 21st Century: A Proposal for Both Developed and Developing Countries", Tax Notes International, pp. 241-255.

Bardhan, A. and Jaffee, D., (2004), "On Intra-Firm Trade and Multinationals: Foreign Outsourcing and Off shoring in Manufacturing", Haas School of Business, University of California, Berkeley.

Bellak, C. and M. Leibrecht (2008), "Do low corporate income tax rates attract FDI? Evidence from central and eastern European countries", forthcoming Applied Economics.

Bartelsman, E.J., Beetsma, R.M.W.J. (2003), "Why pay more? Corporate tax avoidance through transfer pricing in OECD countries", The Journal of Public Economic, Vol. 87 pp.2225-52.

Bernard, J., Weiner, J., (1990), "Multinational Corporations, International Transfer prices and Taxes: Evidence from the U.S. Petroleum Industry", A. Razin and J. Slemord, eds., Taxation in the Global Economy (New York, National Bureau of Economic Research, pp. 123-154.

Bernard, A. B., J. B. Jensen, and P. K. Schott (2006), "Transfer Pricing by U.S.-Based Multinational Firms", NBER Working Paper Series, No. 12493, August 2006, National Bureau of Economic Research, Cambridge.

Borkowski, S. (1997), "The transfer pricing concerns of developed and developing countries", International Journal of Accounting. Vol. 32, No 3, pp 321-36.

Borkowski, S. C. (1997), "Factors motivating transfer pricing choices of Japanese and United States transnational corporations", Journal of International Accounting, Auditing \& Taxation, 6(1), 25-47.

Buettner, Thiess, and G. Wamser, (2007), "Intercompany Loans and Profit Shifting Evidence from Company-Level Data”, CESifo Working Paper No. 1959.

Clausing, Kimberly A, 1998, "The Impact of Transfer Pricing on Intrafirm Trade." NBER Working Paper No. 6688, National Bureau of Economic Research, Cambridge. 
Clausing, Kimberly A., (2003), "Tax-Motivated Transfer Pricing and U.S. Intrafirm Trade Prices", Journal of Public Economics 87, 2207-2223.

Clausing, K.A. (2006), "International tax avoidance and U.S. international trade", National Tax Journal 59, 269-287.

Collins, J.H., D. Kemsley and M. Lang (1998), "Cross-jurisdictional income shifting and earnings valuation", Journal of Accounting Research 36.2, 209-229.

Congress of the United States, 2005, Corporate Income Tax Rates: International Comparisons. Congressional Budget Office (CBO).

De Mooij, R.A. and S. Ederveen (2006), "What a difference does it make? Understanding the empirical literature on taxation and international capital flows", European Commission Economic Papers, 261.

Devereux, M.P. and R. Griffith, (1998), "Taxes and the location of production: evidence from a panel of US multinationals", Journal of Public Economics 68, 335-367.

Devereux, M.P. and R. Griffith, (1999), "The taxation of discrete investment choices", IFS Working Paper 98/16.

Devereux, M.P. and R. Griffith (2002), "The impact of corporate taxation on the location of capital: a review", Economic Analysis \& Policy, Vol. 33, No. 2, pp. 275-292.

Eden, L (2000), "Transfer Pricing, Intrafirm Trade and the BLS International Price Program", US Bureau of Labour Statistics: Washington, DC, BLS Working Paper, 334.

Eden, L; Dacin, M and Wan, W., (2001), "Standards across borders: cross border diffusion of the arm's length standard in North America", Accounting, Organisations and Society, Vol. 26. No 1, pp.1-23.

Edwards, J. and Keen, M., (1996), "Tax competition and leviathan," European Economic Review, Vol. 40.

Elitzur, R.and Mintz, J., (1996), "Transfer pricing rules and corporate tax competition", Journal of Public Economics, Vol. 60, pp. 401-422

Ernest \& Young, (2008), "Precision under pressure global transfer pricing survey, 2007-2008”.http://www.ey.com/Global/assets.nsf/International/EY_Tax_TP Survey_2007/\$file/Tax_TPSurvey_2007.pdf.

Feinschreiber, R., (2004), Transfer Pricing Methods: An Applications Guide, Hoboken, NJ, Wiley. 
Grubert, Harry, and Mutti, John (1991), "Taxes, Tariffs and Transfer Pricing in Multinational Corporate Decision Making," Review of Economics and Statistics, 73(May), pp.285-293.

Gresik, T. A., (2001), "The taxing task of taxing transnationals," Journal of Economic Literature, Vol. 39, pp. 800-838.

Grubert, H., (1995), "Royalties, dividends, and R\&D. In: Proceedings of the Eighty Seventh Annual Conferences on Taxation", National Tax Association, Columbus, $\mathrm{OH}$, pp. 13-24.

Harris, D.G., Morck R., Slemrod J., and Yeung B., (1993), "Income shifting in U.S. multinational corporations", in: Giovannini, A., R.G. Hubbard and J. Slemrod (eds.), Studies in International Taxation. University of Chicago Press, Chicago, pp. 277-302.

Hirshleifer, J. (1956), "On the Economics of Transfer Pricing”. Journal of Business, The University of Chicago, Vol. 29, No. 3 (Jul., 1956), pp. 172-184

Hines, James R. Jr. \& Hubbard, R. Glenn, 1990, "Coming Home to America: Dividend Repatriations by U.S. Multinationals," NBER Working Papers 2931, National Bureau of Economic Research, Inc.

Hines, J., (1994), "Taxes, Technology Transfer, and the R \& D Activities of Multinational Firms". Working paper No. 4932, National Bureau of Economic Research.

Hines, James R., Rice, Eric M., (1994), "Fiscal paradise: foreign tax havens and American business", Quarterly Journal of Economics, 109, 149-182.

Hines Jr., J.R., (1995), "Taxes, technology transfer, and the R\&D activities of multinational firms", In: Feldstein, M., Hines Jr., J.R., Hubbard, R.G. (Eds.), The Effects of Taxation on Multinational Corporations. University of Chicago Press, Chicago, pp. $225-248$.

Hines, J. R. (1996), “Tax Policy and the Activities of Multinational Corporations”, NBER Working Paper No. 5589. Cambridge, National Bureau of Economic Research.

Hines, J. R. (1999), "Lessons from behavioural responses to international taxation", National Tax Journal, June, 305-22.

Horst, T., (1971), "The theory of the multinational firm: optimal behaviour under different tariff and tax rates", Journal of Political Economy 79 (5), 1059-1072.

Huizinga, H., Laeven, L., (2008), "International profit shifting within multinationals: A multi-country perspective”, Journal of Public Economics, Vol. 92 pp. 164-1182. 
Jenkins, G.P., Wright, B.D., (1975), "Taxation of income of multinational corporations: the case of the US petroleum industry", Review of Economics and Statistics 57 (1), 1 -11 .

Klassen, Kenneth, Lang, Mark and Wolfson, Mark, (1993), "Geographic Income Shifting by Multinational Corporations in Response to Tax Rate Changes." Journal of Accounting Research, vol. 31, Studies on International Accounting, pp. 141-173.

Lall, Sanjaya, (1973), "Transfer-pricing by multinational manufacturing firms", Oxford Bulletin of Economics and Statistics, 35(3):173-195.

Leitch, R. A. and Barrett, K. S., (1992), "Multinational transfer pricing: objectives and constraints", Journal of Accounting Literature 11, 47-92.

Matthias Dischinger, (2008), "Profit Shifting by Multinationals and the Ownership Share: Evidence from European Micro Data", Discussion paper 2008-17, University of Munich.

Mintz, J. and A.J. Weichenrieder (2005), "Taxation and the financial structure of German outbound FDI", CESifo Working Paper 1612, Munich.

Newlon, Scott T., (2000), "Transfer pricing and income shifting in integrating economies", In: Cnossen, S. (Ed.). Taxing Capital Income in the European Union: Issues and Options for Reform, Oxford University Press.

Nicodeme, Gaetan, (2001), "Computing Effective Corporate Tax Rates: Comparisons and Results", European Commission, MPRA Paper No. 3808.

OECD (2000), Tax Burdens: Alternative Measures, OECD Tax Policy Studies No. 2, Paris.

Overesch, M., (2006), “Transfer Pricing of Intrafirm Sales as a Profit Shifting Channel Evidence from German Firm Data", ZEW Discussion Paper, No. 06-084, W ZE.

Overesch, M., Wamser, (2008), "The Effects of Company Taxation in EU Accession Countries on German Multinationals", paper prepared for the meeting of the European Tax Policy Forum, London.

Oyelere, P.B., (1998), "International Transfer Pricing and Income Shifting: Evidence from the UK", The European Accounting Review, Vol. 7, issue 4, pp. 623-636.

Plasschaert, S.R.F., (1979), Transfer Pricing and Multinational Corporations: An Overview of Concepts, Mechanisms and Regulations, Saxon House, Westmead, England.

Razin, A., Slemrod, J., (1990), Taxation in the Global Economy, The University of Chicago Press, Ltd., London. 
Rousslang, D.J. (1997), "International income shifting by US multinational corporations", Applied Economics 29, 925-934.

Swenson, Deborah L., (1994), "The impact of U.S. tax reform on foreign direct investment in the United States," Journal of Public Economics, Elsevier, Vol. 54(2), pages 243-266.

Swenson, D.L. (2001), "Tax reforms and evidence of transfer pricing", National Tax Journal, 54, 7-25.

Tax Policy Centre: http://www.taxpolicycenter.org/briefing-book/key-elements/ business/ statutory.cfm

Tyrrall, D., \& Atkinson, M. (1999), International transfer pricing: A practical guide for finance directors. London, Financial Times Management/Pearson Education.

Thomas C. Pearson, (2008), "Proposed International Legal Reforms for Reducing Transfer Pricing Manipulation of Intellectual Property”, 40 N.Y.U. J. Int'L Law \& Politics, 541.

WEICHENRIEDER, A. J., (1996), "Fighting International Tax Avoidance: The Case of Germany”, Institute for Fiscal Studies, Vol. 17, 1, pp. 37-58

Weichenrieder, A. J. (2008), "Profit Shifting in the EU: Evidence from Germany", International Tax and Public Finance, forthcoming.

Zeile, William J., (1997), "U.S. Intrafirm Trade in Goods", Survey of Current Business, 77, 2, pp. 23-38. 
